\title{
Channel Estimation Approach with Low Pilot Overhead in FBMC/OQAM Systems
}

\author{
Jun Sun, ${ }^{1,2}$ Xiaomin Mu, ${ }^{1}$ Dejin Kong $\mathbb{D}^{3}{ }^{3}$ Qian Wang, ${ }^{3}$ Xinmin $\mathrm{Li}^{4}{ }^{4}$ and Xing Cheng ${ }^{5}$ \\ ${ }^{1}$ School of Information Engineering, Zhengzhou University, Zhengzhou 450001, China \\ ${ }^{2}$ School of Electronic and Information, Zhongyuan University of Technology, Zhengzhou 450007, China \\ ${ }^{3}$ State Key Laboratory of New Textile Materials and Advanced Processing Technologies, The School of Electronic and \\ Electrical Engineering, Wuhan Textile University, Wuhan 430200, China \\ ${ }^{4}$ School of Information Engineering, Southwest University of Science and Technology, Mianyang 621010, China \\ ${ }^{5}$ School of Information and Communication Engineering, Beijing Information Science and Technology University, \\ Beijing 100101, China
}

Correspondence should be addressed to Dejin Kong; djkou@wtu.edu.cn

Received 24 February 2021; Revised 27 March 2021; Accepted 16 April 2021; Published 26 April 2021

Academic Editor: Liangtian Wan Copyright $\odot 2021$ Jun Sun et al. This is an open access article distributed under the Creative Commons Attribution License, which
permits unrestricted use, distribution, and reproduction in any medium, provided the original work is properly cited.

\begin{abstract}
In filter bank multicarrier with offset quadrature amplitude modulation (FBMC/OQAM) systems, a large pilot overhead is required due to the existence of the imaginary interference. In this paper, we present an approach to reduce the pilot overhead of channel estimation. A part of pilot overhead is used for transmitting data, and compensating symbols are required and designed to remove the imaginary interference. It is worthwhile to point out that the power of compensating symbols can be helpful for data recovery; hence, the proposed approach decreases the overhead of pilots significantly without the cost of additional pilot energy. In addition, the proposed scheme is extended into multiple input multiple output systems without the performance loss. Compared with the conventional preamble consisting of 3 columns symbols, the pilot overhead is equivalent to 2 column symbols in the proposed preamble. To verify the proposed preamble, numerical simulations are carried out with respects to bit error ratio.
\end{abstract}

\section{Introduction}

Currently, filter bank multicarrier with offset quadrature amplitude modulation (FBMC/OQAM) has been considered as a potential alternative $[1-4]$ to the conventional orthogonal frequency division multiplexing (OFDM). In the past several years, FBMC/OQAM and other filter-based waveforms have been studied to overcome the disadvantages of OFDM. This paper focuses on the FBMC/OQAM technique and presents an effective solution to the channel estimation with low pilot overhead.

Unlike in OFDM, channel estimation in FBMC/OQAM is not a straightforward mission, which is related to that the waveform synthesis and analysis of FBMC/OQAM are not same as that of OFDM. Since the orthogonality condition only is met in real field $[2,5]$, FBMC/OQAM systems transmit real-valued symbols obtained by the real and imaginary parts of complex-valued QAM symbols, and there exists imaginary interferences among the transmitted real-valued symbols, called the intrinsic imaginary interference [6]. For the channel estimation, the imaginary interference has a crucial effect on the pilot design. The imaginary interference has to be eliminated to ensure the good system performance [7]. In particular, while in OFDM receiver data/pilot symbols that are perfectly separated after applying the FFT, the signal samples from the output of the analysis filter in an FBMC/OQAM receiver are subject to intersymbol interference (ISI), both along the time and frequency/subcarriers. ISI-free symbols could be achieved after the channel equalization and the operation of taking the real parts. In the absence of the channel, ISI appears as an imaginary component that adds to the real-valued data symbols which are carried by the FBMC/OQAM waveform, which has to be considered in the pilot design. 
To avert the imaginary interference, a direct method is to disable the symbols surrounding the pilots, which results in a reduced spectral efficiency. By applying the following method, this spectral efficiency loss could be avoid [8]. One dummy symbol is set adjacent to the pilot to eliminate the imaginary interference of the FBMC/OQAM system. This dummy symbol was then called auxiliary pilot (AP) in [9]. Although it is helpful to remove the imaginary interference to pilots, the AP method effectively increases the transmit power. A more complex approach, but more efficient in terms of transmit power, was proposed in [10]. The data symbols surrounding pilots are performed by a linear coding so that the data symbols do not produce any imaginary interference. Nevertheless, to completely remove the imaginary interference, a long code length is required in the coding scheme, which in turn increases a large complexity at both of the transmitter and the receiver. In addition, it also introduces other complications, which are discussed in [11]. In [12], the authors presented the interference approximation method (IAM), in which it was revealed that the channel estimation performance is decided by the so-called pseudopilot power consisting of pilot and the imaginary interference from adjacent data. Afterwards, the modified versions of IAM were presented to improve the pseudopilot power by employing imaginary-valued pilots, i.e., IAM-C [13] and IAM-I [14]. However, it should be noted that the existing IAM-based methods require 3 columns real-valued symbols as pilot overhead to achieve the channel estimation. To reduce the pilot overhead of IAM-based schemes, the iterative algorithm was proposed to eliminate the imaginary interference for channel estimation [15]. Nevertheless, it suffers from the problems of high computational complexity. In [16], the authors proposed the pairs of the real pilots (POP) method, with only 2 columns pilots. However, by reason of the bad ability of against noise, the POP method exhibits poor channel estimation performance compared with the conventional IAM-based methods.

In this paper, we present an approach to reduce the pilot overhead of channel estimation in FBMC/OQAM systems. Compared with the conventional pilot structures, a part of pilot overhead is used for transmitting data, and compensating symbols are required and designed to eliminate the imaginary interference from data. Note that it is proven that the power of compensating symbols can be helpful for data recovery; hence, the proposed approach decreases the overhead of pilots significantly without the cost of additional pilot energy. Compared with the conventional methods with 3 columns pilots, the proposed scheme only requires 2 columns pilots for the channel estimation. In addition, the proposed approach is extended into multiple input multiple output- (MIMO-) based FBMC (MIMOFBMC) systems.

The rest of this paper is organized as follows. The IAM method is briefly introduced in Section 2. The proposed approach is presented in Section 3, followed by the corresponding algorithm. Then, the proposed scheme is extended into MIMO-FBMC systems in Section 4. Section 5 shows the simulations, and Section 6 is the conclusions.

\section{Channel Estimation with the IAM Method in FBMC/OQAM Systems}

2.1. System Model. As depicted in Figure 1, the diagram block of FBMC/OQAM transceiver is presented, in which $M$ subcarriers are considered in the FBMC/OQAM system with the subcarrier spacing $1 / M$. Note that the complex-valued data symbols have the interval of $M$ samples in time, and by partitioning each complex-valued symbol, a pair of PAM symbols are obtained. The PAM symbols are denoted by $d_{m, n}$, with $m$ the subchannel index and $n$ the time index. In addition, $d_{m, 2 \bar{n}}$ and $d_{m, 2 \bar{n}+1}$, i.e., the real and imaginary parts of a QAM symbol, have the interval of $M / 2$ samples in time. $g[l]$ is the prototype filter and spans over the time interval 0 $\leq l \leq K M-1$, where $K$ is the overlapping factor and is supposed to be a positive integer. It is further assumed that the filter $g[l]$ is even and symmetric around its center, hence, $g[l]=g[K M-1-l]$ for $0 \leq l \leq K M-1$.

Following Figure 1, the transmitted signal is [17]

$$
s[l]=\sum_{m=0}^{M-1} \sum_{n \in \mathbb{Z}} d_{m, n} g\left[l-n \frac{M}{2}\right] e^{j 2 \pi m l / M} e^{j \pi(m+n) / 2},
$$

where $M$ stands for the subcarrier number. $d_{m, n}$ represents one transmitted symbol of position $(m, n)$, with only real value.

Then, the signal at the receive antenna is obtained

$$
r[k]=h[l] * s[l]+\eta[l]
$$

where the $\operatorname{sign} *$ is the convolution operator. $h[l]$ represents the multipath channel, and there exists a channel noise $\eta[l]$, satisfying the Gaussian distribution with variance $\sigma^{2}[18-20]$.

Then, demodulations at the receiver can be written as

$$
\widehat{d}_{m, n}=\sum_{l=-\infty}^{\infty} r[l] g\left[l-n \frac{M}{2}\right] e^{-j 2 \pi m l / M} e^{-j \pi(m+n) / 2} .
$$

Then, an operator of taking real part is required.

$$
\Re\left\{\widehat{d}_{m, n}\right\}=d_{m, n}
$$

Note that channel estimation is necessary in the FBMC/OQAM system under the multipath channel.

2.2. The IAM Method. In [12], the IAM method with 3 column pilots has been presented for the channel estimation in FBMC/OQAM systems. The estimation model of IAM can be obtained [12]:

$$
\widehat{d}_{m, n} \approx h_{m, n}\left(d_{m, n}+d_{m, n}^{(*)}\right)+\eta_{m, n}
$$

where $\widehat{d}_{m, n}$ is the demodulation at the receiver of FBMC/OQAM. $h_{m, n}$ stands for frequency-domain channel at $m$-th subcarrier, which is supposed quasi-invariant in the time domain in this paper, i.e., 


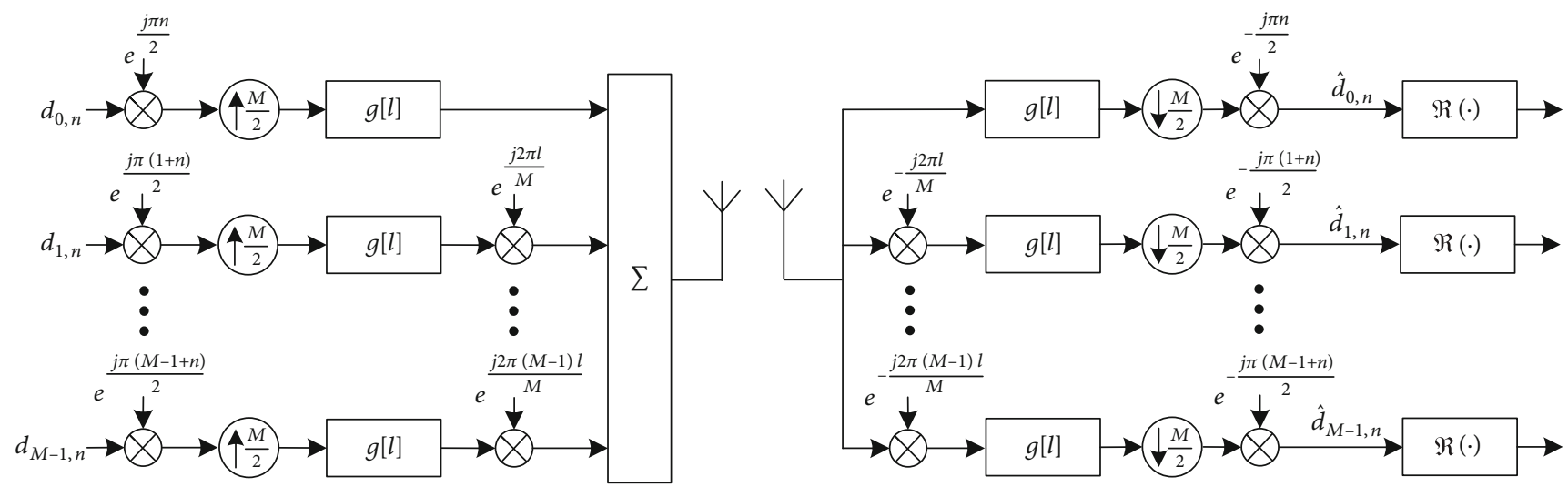

Figure 1: The FBMC/OQAM system diagram.

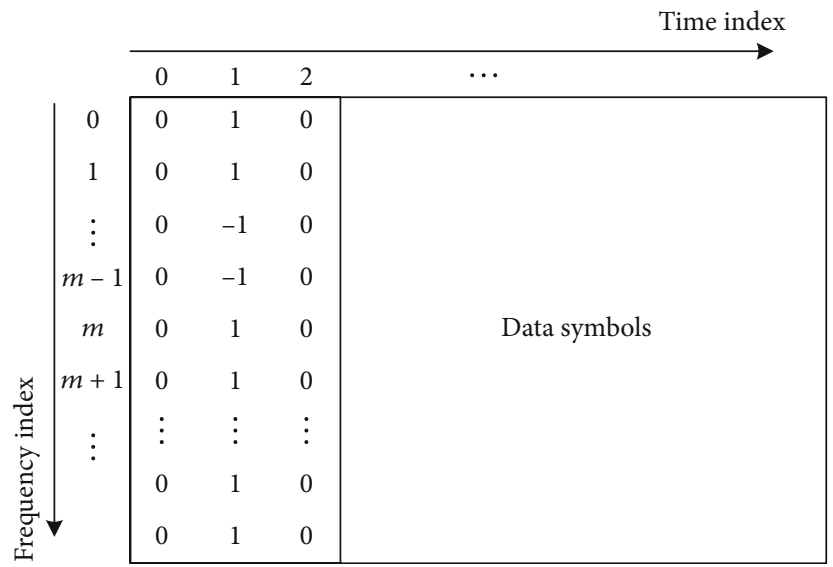

(a) The conventional 3-column preamble structure of IAM

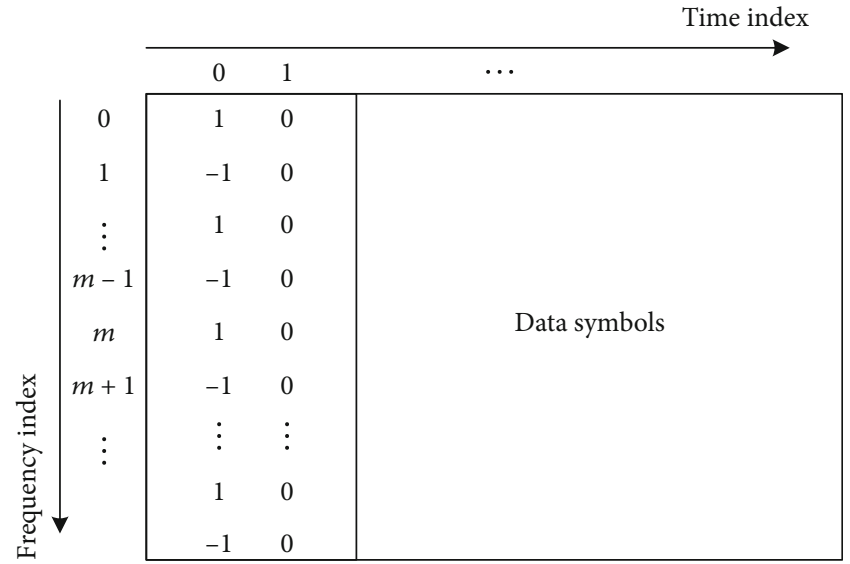

(b) The preamble structure of POP

FIgURE 2: The conventional preamble and the proposed preamble in FBMC/OQAM.

$$
h_{m, n} \approx h_{m, 0}=\sum_{k=0}^{L-1} h[k] e^{-2 j \pi m k / M}
$$

with the maximum channel delay spread of $L$. The imaginary interference $d_{m, n}^{(*)}$ is written as

$$
d_{m, n}^{(*)}=\sum_{\Omega} d_{m, n} \xi_{m+p, n+q}^{m, n}
$$

with $\Omega=\{(p, q)|| p|| q \mid, \leq \operatorname{land}(p, q) \neq(0,0)\}$, and the imaginary interference factor, $\xi_{m+p, n+q}^{m, n}$, is defined as

$$
\xi_{m+p, n+q}^{m, n}=\xi_{p, q}^{0,0}=\sum_{l=-\infty}^{\infty} g[l] g\left[l+q \frac{M}{2}\right] e^{j 2 \pi p l / M} e^{j \pi(p+q) / 2} .
$$

And the noise term $\eta_{m, n}$ is

$$
\eta_{m, n}=\sum_{l=-\infty}^{\infty} \eta[l] g\left[l-n \frac{M}{2}\right] e^{-j 2 \pi m l / M} e^{-j \pi(m+n) / 2} .
$$

Then, the IAM channel estimation is written as [12]

$$
\widehat{h}_{m, n}=\frac{\widehat{d}_{m, n}}{d_{m, n}+d_{m, n}^{(*)}}=h_{m, n}+\frac{\eta_{m, n}}{d_{m, n}+d_{m, n}^{(*)}} .
$$

Figure 2(a) depicts the existing preamble of IAM, i.e., $d_{m, 0}=d_{m, 2}=0$ with $m=0,1, \cdots, M-1, \quad d_{4 l, 1}=d_{4 l+1,1}=1$, and $d_{4 l+2,1}=d_{4 l+3,1}=-1$ with $l=0,1, \cdots, M / 4-1$. Note that it is well known that the interval of an FBMC/OQAM symbol is only half of that of an OFDM symbol. Therefore, the pilot overhead in FBMC/OQAM systems is 1.5 times of that classical OFDM systems.

2.3. POP Method. The POP method is another preamblebased channel estimation method in [16]. As shown in Figure 2(b), only two columns of real-valued pilots are required in the POP method, i.e., $d_{2 k, 0}=1, d_{2 k+1,1}=-1$ with $k=0,1, \cdots, M / 2-1$, and $d_{m, 1}=0$ with $m=0,1, \cdots, M-1$.

Suppose $\left(m_{1}, n_{1}\right),\left(m_{2}, n_{2}\right)$ is the time-frequency positions of two symbols. The channel estimation by POP can be written as 


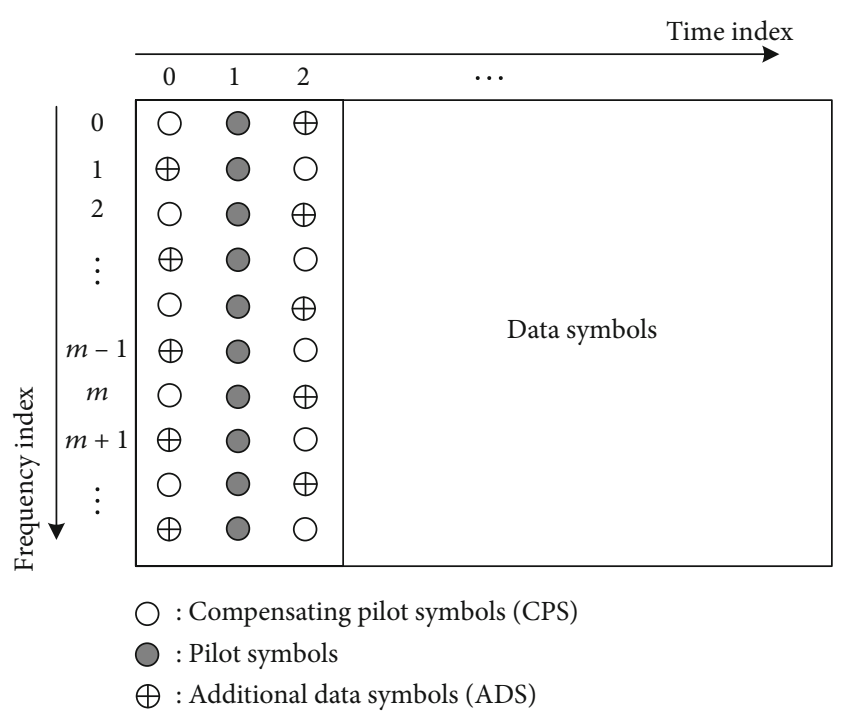

Figure 3: The proposed pilot structure in FBMC/OQAM.

$$
\left\{\begin{array}{l}
\mathfrak{R}\left(h_{m_{1}, n_{1}}\right)=\frac{\mathfrak{R}\left(\hat{d}_{m_{1}, n_{1}}\right)+C \cdot \mathfrak{I}\left(\hat{d}_{m_{1}, n_{1}}\right)}{d_{m_{1}, n_{1}}}, \\
\mathfrak{J}\left(h_{m_{1}, n_{1}}\right)=C \cdot \mathfrak{R}\left(h_{m_{1}, n_{1}}\right),
\end{array}\right.
$$

where $\mathfrak{R}(\cdot)$ and $\mathfrak{\Im}(\cdot)$ are the operators of taking the real part and the imaginary part, respectively. $C$ is the ratio between the imaginary part and the real part of $h_{m_{1}, n_{1}}$,

$$
C=\frac{d_{m_{2}, n_{2}} \cdot \mathfrak{R}\left(\hat{d}_{m_{1}, n_{1}}\right)-d_{m_{1}, n_{1}} \cdot \mathfrak{R}\left(\hat{d}_{m_{2}, n_{2}}\right)}{d_{m_{1}, n_{1}} \cdot \mathfrak{I}\left(\hat{d}_{m_{2}, n_{2}}\right)-d_{m_{2}, n_{2}} \cdot \mathfrak{J}\left(\widehat{d}_{m_{1}, n_{1}}\right)} .
$$

Then, the channel coefficients have been estimated. This approach does not require any knowledge of the prototype function and could be adopted as preamble-based method and also as a scattered-based channel estimation method.

\section{Proposed Channel Estimation Scheme for FBMC/OQAM Systems}

3.1. Preamble Structure of the Proposed Scheme. Figure 3 depicts the proposed preamble structure, where $d_{m, 1}, m=0$, $1, \cdots, M-1$ are pilot symbols. Different from Figure 2(a), a part time-frequency resources of the first and the third columns are used to transmit additional data symbols (ADS), i.e., $\quad \mathbf{u}=\left[u_{0}, x_{1}, \cdots, u_{M-1}\right]^{T}$ with $u_{2 m}=a_{2 m, 2}$ and $u_{2 m+1}=$ $a_{2 m+1,0}$. To eliminate the imaginary interference, compensating pilot symbols (CPS) are required, i.e., $\mathbf{v}=$ $\left[v_{0}, v_{1}, \cdots, v_{M-1}\right]^{T}$ with $v_{2 m}=a_{2 m, 0}$ and $v_{2 m+1}=a_{2 m+1,2}$. According to the criteria that the imaginary interferences from ADS and CPS should be mutually canceling, $\mathbf{v}$ can be designed by

$$
\boldsymbol{\Phi} H u+\boldsymbol{\Omega} H v=0,
$$

where $\mathbf{H}$ represents the diagonal matrix with $m$-th diagonal element $h_{m, 0}$, and

$$
\begin{gathered}
\boldsymbol{\Phi}=\left(\begin{array}{ccccc}
\xi_{0,2}^{0,1} & \xi_{1,0}^{0,1} & \xi_{2,2}^{0,1} & \cdots & \xi_{M-1,0}^{0,1} \\
\xi_{0,2}^{1,1} & \xi_{1,0}^{1,1} & \xi_{2,2}^{1,1} & \cdots & \xi_{M-1,0}^{1,1} \\
\xi_{0,2}^{2,1} & \xi_{1,0}^{2,1} & \xi_{2,2}^{2,1} & \cdots & \xi_{M-1,0}^{2,1} \\
\vdots & \vdots & \vdots & \ddots & \vdots \\
\xi_{0,2}^{M-1,1} & \xi_{1,0}^{M-1,1} & \xi_{2,2}^{M-1,1} & \ldots & \xi_{M-1,0}^{M-1,1} \\
& & & &
\end{array}\right), \\
\boldsymbol{\Omega}=\left(\begin{array}{cccccc}
\xi_{0,0}^{0,1} & \xi_{1,2}^{0,1} & \xi_{0,2}^{0,1} & \cdots & \xi_{M-1,2}^{0,1} \\
\xi_{0,0}^{1,1} & \xi_{1,2}^{1,1} & \xi_{2,0}^{1,1} & \cdots & \xi_{M-1,2}^{1,1} \\
\xi_{0,0}^{2,1} & \xi_{1,2}^{2,1} & \xi_{2,0}^{2,1} & \cdots & \xi_{M-1,2}^{2,1} \\
\vdots & \vdots & \vdots & \ddots & \vdots \\
\xi_{0,0}^{M-1,1} & \xi_{1,2}^{M-1,1} & \xi_{2,0}^{M-1,1} & \cdots & \xi_{M-1,2}^{M-1,1} \\
& & & &
\end{array}\right) .
\end{gathered}
$$

Then, it is obtained as

$$
v=-H^{-1} \mathbf{\Omega}^{-1} \Phi H u \text {. }
$$

It should be noted that (16) cannot be used to design CPS directly due to the fact that it is difficult to obtain $H$ at the transmitter.

It is proven that $\boldsymbol{\Omega}^{-1} \boldsymbol{\Phi}$ is a unitary matrix in the appendix. Let $\alpha_{m n}$ be the $(m, n)$-th entry of $\boldsymbol{\Omega}^{-1} \boldsymbol{\Phi}$, and it can be obtained as

$$
\left\{\begin{array}{l}
\sum_{n=0}^{M-1}\left|\alpha_{m n}\right|^{2}=1, \\
\sum_{n}\left|\alpha_{m n}\right|^{2} \approx 0.9991,|m-n| \leq 3,|m-n| \geq M-3 .
\end{array}\right.
$$

Thus, it can be concluded that $\alpha_{m n} \approx 0$ for $M-3>\mid m-$ $n \mid>3$.

In addition, define $C=H^{-1} \boldsymbol{\Omega}^{-1} \boldsymbol{\Phi} H$, and its $(m, n)$-th element is $C_{m n}=\left(h_{m, 0} / h_{n, 0}\right) \beta_{m n}$, which is close to zero for $M$ $-3>|m-n|>3$. Then, it can be assumed that $H_{m, 0} / H_{n, 0} \approx$ 1 for $|m-n| \leq 3$ or $|m-n| \geq M-3$. Therefore, $C_{m n} \approx \beta_{m n}$ and $v$ can be obtained by

$$
v=-\Omega^{-1} \Phi u
$$

3.2. Data Recovery of ADS. In this subsection, it is proven that the CPS is helpful for data recovery of ADS. Therefore, the ADS has the similar ability to fight against the noise compared with data symbols as we can see below. 


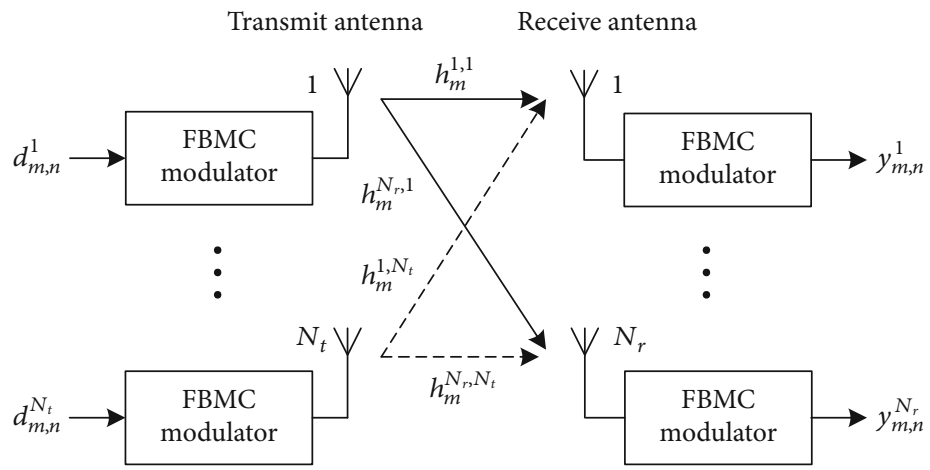

FIgUre 4: The MIMO-FBMC system model.

\begin{tabular}{ccccccc}
\multicolumn{3}{c}{ Antenna 1 } & \multicolumn{3}{c}{ Antenna 2 } \\
0 & 1 & 0 & 0 & 0 & 0 \\
0 & 0 & 0 & 0 & 0 & 0 \\
0 & 0 & 0 & 0 & 1 & 0 \\
0 & 0 & 0 & 0 & 0 & 0 \\
0 & -1 & 0 & 0 & 0 & 0 \\
$\vdots$ & $\vdots$ & $\vdots$ & $\vdots$ & $\vdots$ & $\vdots$ \\
0 & 0 & 0 & 0 & 0 & 0
\end{tabular}

(a) Conventional preamble structure in MIMO-FBMC

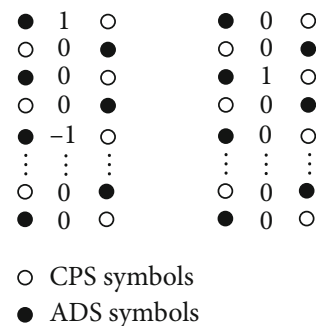

(b) Proposed preamble structure in MIMO-FBMC

Figure 5: Proposed preamble in MIMO-FBMC systems.

According to (5), the demodulation of $v$ and $u$ is

$$
\left\{\begin{array}{l}
u=H\left(u+u^{(*)}\right)+\boldsymbol{\eta}^{u}, \\
v=H\left(v+v^{(*)}\right)+\boldsymbol{\eta}^{v}
\end{array}\right.
$$

where $u^{(*)}$ and $v^{(*)}$ are the imaginary interference, respectively, i.e., $\mathbf{u}^{(*)}=\left[u_{0}^{(*)}, u_{1}^{(*)}, \cdots, u_{M-1}^{(*)}\right]^{T}$, in which $u_{2 k}^{(*)}=$ $d_{2 k, 2}^{(*)}$ and $u_{2 k+1}^{(*)}=d_{2 k+1,0}^{(*)}, \mathbf{v}^{(*)}=\left[v_{0}^{(*)}, v_{1}^{(*)}, \cdots, v_{M-1}^{(*)}\right]^{T}$, in which $v_{2 k}^{(*)}=d_{2 k, 0}^{(*)}$ and $v_{2 k+1}^{(*)}=d_{2 k+1,2}^{(*)} \cdot \boldsymbol{\eta}^{u}=\left[\eta_{0}^{u}, \eta_{1}^{u}, \eta_{3}^{u}, \cdots, \eta_{M-1}^{u}\right]^{T}$ with $\eta_{2 i}^{u}=\eta_{2 i, 2}$ and $\eta_{2 i+1}^{u}=\eta_{2 i+1,0} . \boldsymbol{\eta}^{v}=\left[\eta_{0}^{v}, \eta_{1}^{v}, \eta_{2}^{v}, \cdots, \eta_{M-1}^{v}\right]^{T}$ with $\eta_{2 i}^{v}=\eta_{2 i, 0}$ and $\eta_{2 i+1}^{v}=\eta_{2 i+1,1}$.

According to (18), we have

$$
u=\left(-\boldsymbol{\Omega}^{-1} \boldsymbol{\Phi}\right)^{-1} v
$$

Let $u=\left(-\boldsymbol{\Omega}^{-1} \boldsymbol{\Phi}\right)^{-1} v$, and it is obtained

$$
\begin{aligned}
u & =\left(-\boldsymbol{\Omega}^{-1} \boldsymbol{\Phi}\right)^{-1} H\left(v+v^{(*)}\right)+\left(-\boldsymbol{\Omega}^{-1} \boldsymbol{\Phi}\right)^{-1} \boldsymbol{\eta}^{v} \\
& =\left(-\boldsymbol{\Omega}^{-1} \boldsymbol{\Phi}\right)^{-1} H\left(v+v^{(*)}\right)+\tilde{\boldsymbol{\eta}} .
\end{aligned}
$$

When the channel noise vanishes, we have $\mathfrak{R}\left(H^{-1} u\right) \approx$ $\left(-\boldsymbol{\Omega}^{-1} \boldsymbol{\Phi}\right)^{-1} v=u=\mathfrak{R}\left(H^{-1} u\right)$. Then, $1 / 2 u+1 / 2 u$ will be the input of channel equalizer instead of $u$, since the noise variance will be reduced half after the linear combination. By this way, the ADS has similar capability to fight against the noise compared with the data symbols.

\section{Channel Estimation in MIMO-FBMC}

In this section, the proposed scheme in Section 3 can be easily extended into MIMO-FBMC systems with $N_{t}$ transmit antennas and $N_{r}$ receive antennas, as shown in Figure 4. Denote the symbol of the $t$-th transmit antenna as $d_{m, n}^{t}$, and let $h_{m}^{r, t}$ be the channel frequency response between the $r$ -th receive antenna and the $t$-th transmit antenna at the $m$ -th subcarrier.

Without loss of generality, $N_{t}$ is set to 2 in this section for simplicity. As is well known, the imaginary interference exists among the FBMC/OQAM symbols [12]. When there exists the imaginary interference between different antennas, it is difficult for one user to perform the channel estimation since the imaginary interference from other users is not available. Therefore, the key of the preamble design in MIMOFBMC systems is the imaginary interference cancelation between antennas. Figure 5(a) depicts the conventional preambles on the two transmit antennas in MIMO-FBMC systems. Zeros are placed in the first and third columns to avert imaginary interference from data. It should be noted that nonzero pilots only locate in a part of subcarriers to ensure no imaginary interference between antennas, i.e., $d_{4 p}$ , $p=0,1, \cdots, M / 4-1$ is a nonzero pilot for the first transmit antenna, and $d_{4 p+2}, p=0,1, \cdots, M / 4-1$ is a nonzero pilot for the second transmit antenna. In our proposed preambles, in Figure 5(b), the nonzero pilots in the second column are 


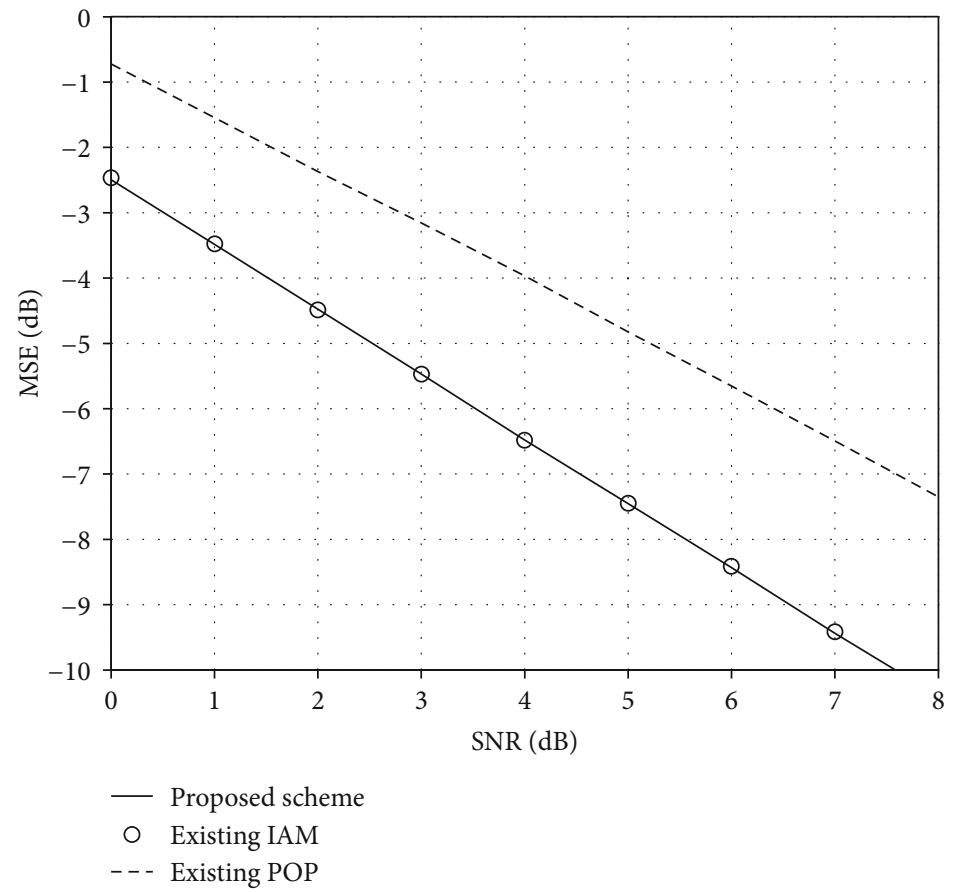

FIgURE 6: MSE of the proposed scheme.

the same as the conventional preambles. Differently, half of subcarriers in the first and third columns are placed by data symbols, improving the spectral efficiency. To eliminate the imaginary interference, compensating pilot symbols are required and designed according to (16) in Section 3. Therefore, compared with the conventional preambles, the pilot overhead of the proposed preamble is reduced by $1 / 3$.

At the receiver, the demodulation of the $r$-th receive antenna is obtained

$$
y_{m_{0}, n_{0}}^{r}=\sum_{t=1}^{N_{t}}[h_{m_{0}}^{r, t} d_{m_{0}, n_{0}}^{t}+\underbrace{\sum h_{m}^{r, t} d_{m, n}^{t} \xi_{m, n}^{m_{0}, n_{0}}}_{I_{m_{0}, n_{0}}^{t}}]+\eta_{m_{0}, n_{0}}^{r},
$$

where noise $\eta_{m_{0}, n_{0}}^{r}$ satisfies the Gaussian distribution with mean 0 and variance $\sigma^{2} . I_{m_{0}, n_{0}}^{t}$ is the imaginary interference term to the transmit symbol $d_{m_{0}, n_{0}}^{t}$.

As mentioned above, the imaginary interference between antennas can be removed completely. Then, for the proposed preambles in Figure 5(b), equation (22) can be rewritten as

$$
\begin{aligned}
y_{4 p, 1}^{r} & =h_{4 p}^{r, 1} d_{4 p, 1}^{1}+\eta_{4 p, 1}^{r}, \\
y_{4 p+2,1}^{r} & =h_{4 p+2}^{r, 2} d_{4 p+2,1}^{2}+\eta_{4 p+2,1}^{r}, \\
p & =0,1, \cdots, M / 4-1 .
\end{aligned}
$$

Accordingly, the channel estimation in MIMO-FBMC systems can be obtained as

$$
\begin{aligned}
h_{4 p}^{r, 1} & =\frac{y_{4 p, 1}^{r}}{d_{4 p, 1}^{1}}, \\
h_{4 p+2}^{r, 2} & =\frac{y_{4 p+2,1}^{r}}{d_{4 p+2,1}^{2}}, p=0,1, \cdots, M / 4-1 .
\end{aligned}
$$

Then, the simple linear interpolation is performed on $h_{4 p}^{r, 1}$ and $h_{4 p+2}^{r, 2}$ to obtain the channel estimation of all subcarriers, respectively. It should be noted that our proposed approach could decrease the overhead of pilots significantly without the cost of additional pilot energy. Although the CPS symbols consume energy, it will be completely used for symbol recovery as presented in Subsection 3.2.

\section{Simulation Results}

In this section, we evaluate the performance of the proposed channel estimation approaches that are presented in this paper through computer simulations. The FBMC/OQAM system employs the PHYDYAS filter [17] and the overlap parameter $K=4$. The multipath channel model is simulated, i.e., SUI proposed by the IEEE 802.16 broadband wireless access working group [21]. In simulations, the following parameters are considered.

(i) Subcarrier number: 2048

(ii) Sampling rate $(\mathrm{MHz}): 30.72$

(iii) Path number: 3

(iv) Delay of path $(\mu s): 0,0,4,0.9$ 


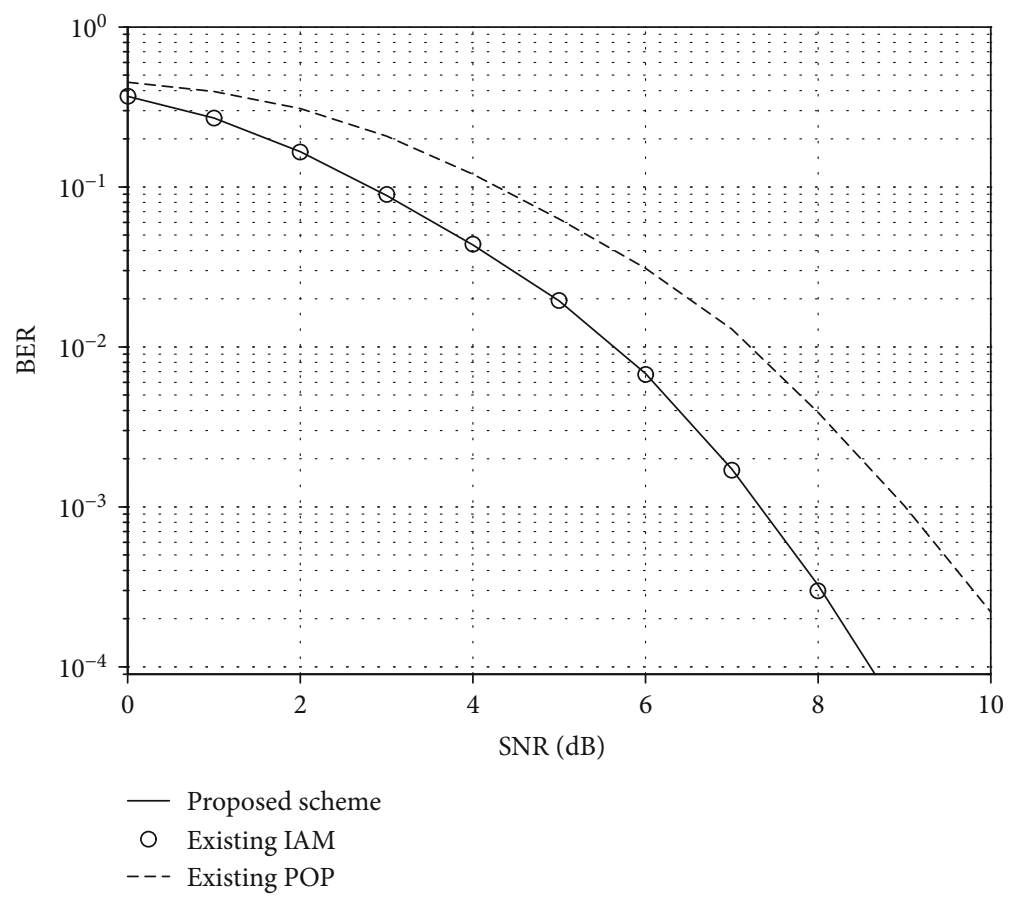

FIGURE 7: BER of the proposed scheme.

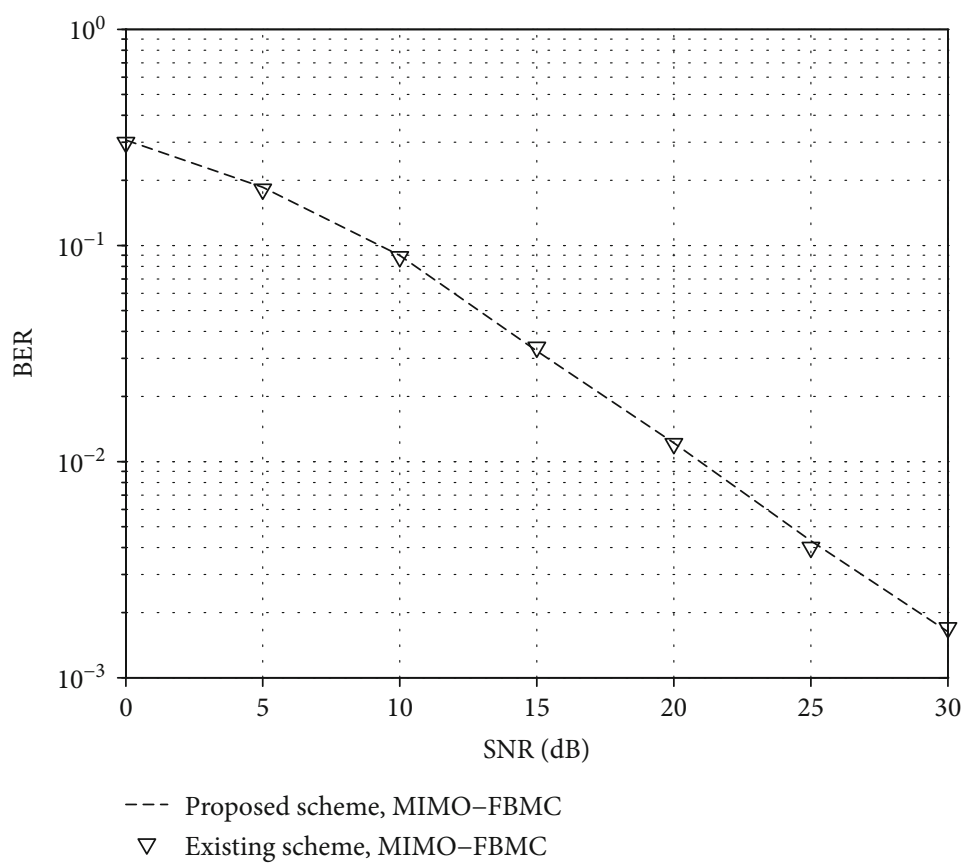

FIGURE 8: BER of the proposed scheme in MIMO-FBMC with 2 transmit antennas and 2 receive antennas.

(v) Power delay profile $(\mathrm{dB}): 0,-5,-10$

(vi) Modulation: 4QAM

(vii) Channel coding: convolutional coding [12]

For comparison, we also give the performance of POP [16], which only requires two columns of pilots.
Figures 6 and 7 show the MSE and BER performances of the proposed approach. It can be easily seen that obvious performance loss is observed in the conventional POP method. As presented in [16], the performance of POP depends on a random power that could be close to zero sometimes, leading to a poor performance. In addition, the proposed scheme can achieve the same MSE and BER 
performances as the IAM method, which indicates that the imaginary interference between pilots and data symbols can be eliminated completely in the proposed scheme. It should be noted that only two columns pilots are needed in the proposed scheme, while the conventional preamble requires three columns of pilots. Therefore, better spectral efficiency can be achieved by our proposed channel estimation approach.

Figure 8 shows BER of the proposed approach in MIMO-FBMC systems, in which both of $N_{t}$ and $N_{r}$ are set to 2. The proposed 2-column preamble can achieve similar BER compared to the conventional 3-column preamble, which demonstrates the effectiveness of the proposed scheme. It should be noted that our proposed scheme can reduce the pilot overhead significantly without the cost of additional pilot energy. Although the CPS symbols consume energy as shown in Figures 3 and 5, it will be completely used for symbol recovery as presented in Subsection 3.2.

\section{Conclusions}

In this paper, an approach was presented for the pilot overhead reduction in the channel estimation of FBMC/OQAM systems. Compared with the conventional methods with 3 columns pilots, the proposed scheme only requires 2 column pilots. In addition, the proposed approach was also extended into MIMO-FBMC systems. It was also proven that our proposed approach could decrease the overhead of pilots significantly without the cost of additional pilot energy and performance loss. Simulations have been done to verify the effectiveness of the proposed approach.

\section{Appendix}

From (8), we have $\zeta_{0,1}^{0,0}=-\zeta_{0,-1}^{0,0}=g$ and $\zeta_{1,1}^{0,0}=\zeta_{1,-1}^{0,0}=\zeta_{-1,1}^{0,0}$ $=\zeta_{-1,-1}^{0,0}=\zeta_{M-1,-1}^{0,0}=\zeta_{-M+1,1}^{0,0}=f$ where $g, f$ are imaginaryvalued and constant. Furthermore, when $M-1>|p|>1$ or $|q|>1, \zeta_{m+p, n+q}^{m, n} \approx 0$ [12]. Thus, (14) and (15) can be rewritten as

$$
\boldsymbol{\Phi}=\left(\begin{array}{cccccccc}
g & f & 0 & 0 & 0 & \cdots & 0 & f \\
f & -g & f & 0 & 0 & \cdots & 0 & 0 \\
0 & f & g & f & 0 & \cdots & 0 & 0 \\
0 & 0 & f & -g & f & \cdots & 0 & 0 \\
0 & 0 & 0 & f & g & \cdots & 0 & 0 \\
\vdots & \vdots & \vdots & \vdots & \vdots & \ddots & \vdots & \vdots \\
0 & 0 & 0 & 0 & 0 & \cdots & g & f \\
f & 0 & 0 & 0 & 0 & \cdots & f & -g \\
& & & & & & &
\end{array}\right),
$$

$$
\boldsymbol{\Omega}=\left(\begin{array}{cccccccc}
-g & f & 0 & 0 & 0 & \cdots & 0 & f \\
f & g & f & 0 & 0 & \cdots & 0 & 0 \\
0 & f & -g & f & 0 & \cdots & 0 & 0 \\
0 & 0 & f & g & f & \cdots & 0 & 0 \\
0 & 0 & 0 & f & -g & \cdots & 0 & 0 \\
\vdots & \vdots & \vdots & \vdots & \vdots & \ddots & \vdots & \vdots \\
0 & 0 & 0 & 0 & 0 & \cdots & -g & f \\
f & 0 & 0 & 0 & 0 & \cdots & f & g \\
& & & & & & &
\end{array}\right),
$$

respectively.

In addition, let

$$
G=\left(\begin{array}{cccccccc}
g & 0 & 0 & 0 & 0 & \cdots & 0 & 0 \\
0 & -g & 0 & 0 & 0 & \cdots & 0 & 0 \\
0 & 0 & g & 0 & 0 & \cdots & 0 & 0 \\
0 & 0 & 0 & -g & 0 & \cdots & 0 & 0 \\
0 & 0 & 0 & 0 & g & \cdots & 0 & 0 \\
\vdots & \vdots & \vdots & \vdots & \vdots & \ddots & \vdots & \vdots \\
0 & 0 & 0 & 0 & 0 & \cdots & g & 0 \\
0 & 0 & 0 & 0 & 0 & \cdots & 0 & -g \\
& & & & & & &
\end{array}\right),
$$

denote an $M \times M$ diagonal matrix and $F=\boldsymbol{\Phi}-G$. Therefore, we have

$$
\left\{\begin{array}{l}
\Phi=F+G, \\
\boldsymbol{\Omega}=F-G .
\end{array}\right.
$$

Note that $G^{\dagger}=-G$ and $F^{\dagger}=-F$ since both of $g$ and $f$ are imaginary-valued, where $(\cdot)^{\dagger}$ is the Hermitian transpose operation. For the even subcarrier $M$, it can be easily obtained $F G+G F=0$ where 0 is the zero matrix. Then, we have

$$
\begin{aligned}
F F+G G & =F F+G G, \\
F F+G G+F G+G F & =F F+G G-F G-G F, \\
(F+G)(F+G) & =(F-G)(F-G), \\
(F+G)(F-G)^{-1} & =(F+G)^{-1}(F-G), \\
\Phi \boldsymbol{\Omega}^{-1} & =\boldsymbol{\Phi}^{-1} \boldsymbol{\Omega} .
\end{aligned}
$$

Based on the equations $\left(\boldsymbol{\Omega}^{-1} \boldsymbol{\Phi}\right)^{\dagger}=\boldsymbol{\Phi}^{\dagger}\left(\boldsymbol{\Omega}^{\dagger}\right)^{-1}=\boldsymbol{\Phi} \boldsymbol{\Omega}^{-1}$ and $\left(\boldsymbol{\Omega}^{-1} \boldsymbol{\Phi}\right)^{-1}=\boldsymbol{\Phi}^{-1} \boldsymbol{\Omega}$, we have

$$
\left(\boldsymbol{\Omega}^{-1} \boldsymbol{\Phi}\right)^{\dagger}=\left(\boldsymbol{\Omega}^{-1} \boldsymbol{\Phi}\right)^{-1}
$$


Then,

$$
\left(\boldsymbol{\Omega}^{-1} \boldsymbol{\Phi}\right)^{\dagger}\left(\boldsymbol{\Omega}^{-1} \boldsymbol{\Phi}\right)=\left(\boldsymbol{\Omega}^{-1} \boldsymbol{\Phi}\right)\left(\boldsymbol{\Omega}^{-1} \boldsymbol{\Phi}\right)^{\dagger}=I .
$$

Finally, we can obtain that $\mathbf{\Omega}^{-1} \boldsymbol{\Phi}$ is a unitary matrix.

\section{Data Availability}

The data used to support the findings of this study are available from the corresponding author upon request.

\section{Conflicts of Interest}

The authors declare that there are no conflicts of interest regarding the publication of this paper.

\section{Acknowledgments}

This work was financially supported in part by the National Science Foundation of China with Grant number 62001333, Nature Science Foundation of Southwest University of Science and Technology with Grant number 18zx7142, and the Scientific Research Fund of Beijing Information Science and Technology University with Grant number 2025018.

\section{References}

[1] D. Kong, D. Qu, and T. Jiang, "Time domain channel estimation for OQAM-OFDM systems: algorithms and performance bounds," IEEE Transactions on Signal Processing, vol. 68, no. 2 , pp. 322-330, 2014.

[2] P. Siohan, C. Siclet, and N. Lacaille, "Analysis and design of OFDM/OQAM systems based on filterbank theory," IEEE Transactions on Signal Processing, vol. 50, no. 5, pp. 11701183, 2002.

[3] R. Nissel and M. Rupp, "Pruned DFT-spread FBMC: low PAPR, low latency, high spectral efficiency," IEEE Transactions on Communications, vol. 66, no. 10, pp. 4811-4825, 2018.

[4] R. Zakaria, D. Silva, and D. le Ruyet, "Lattice-reduction-aided equalization for MIMO-FBMC systems," IEEE Communications Letters, vol. 8, no. 1, pp. 101-104, 2019.

[5] H. Wang, "Low-Complexity MIMO-FBMC sparse channel parameter estimation for industrial big data communications," IEEE Transactions on Industrial Informatics, vol. 17, no. 5, pp. 3422-3430, 2021.

[6] D. Kong, J. Li, K. Luo, and T. Jiang, "Reducing pilot overhead: channel estimation with symbol repetition in MIMO-FBMC systems," IEEE Transactions on Communications, vol. 68, no. 12, pp. 7634-7646, 2020.

[7] S. Hu, Z. Liu, Y. L. Guan, C. Jin, Y. Huang, and J. M. Wu, "Training sequence design for efficient channel estimation in MIMO-FBMC systems," IEEE Access, vol. 5, pp. 4747-4758, 2017.

[8] J. P. Javaudin, D. Lacroix, and A. Rouxel, "Pilot-aided channel estimation for OFDM/OQAM," in The 57th IEEE Semiannual Vehicular Technology Conference, 2003. VTC 2003-Spring, pp. 1581-1585, Jeju, Korea (South), April 2003.

[9] T. H. Stitz, T. Ihalainen, A. Viholainen, and M. Renfors, "Pilotbased synchronization and equalization in filter bank multicarrier communications," EURASIP Journal on Advances in Signal Processing, vol. 2010, no. 1, 2010.
[10] C. Lele, R. Legouable, and P. Siohan, "Channel estimation with scattered pilots in OFDM/OQAM," in 2008 IEEE 9th Workshop on Signal Processing Advances in Wireless Communications, pp. 286-290, Recife, Brazil, July 2008.

[11] W. Cui, D. Qu, T. Jiang, and B. Farhang-Boroujeny, "Coded auxiliary pilots for channel estimation in FBMC-OQAM systems," IEEE Transactions on Vehicular Technology, vol. 65, no. 5, pp. 2936-2946, 2016.

[12] C. Lélé, J. P. Javaudin, R. Legouable, A. Skrzypczak, and P. Siohan, "Channel estimation methods for preamble-based OFDM/OQAM modulations," European Transactions on Telecommunications, vol. 19, no. 7, pp. 741-750, 2008.

[13] J. Du and S. Signell, "Novel preamble-based channel estimation for OFDM/OQAM systems," in 2009 IEEE International Conference on Communications, Dresden, Germany, June 2009.

[14] C. Lélé, P. Siohan, and R. Legouable, " 2 dB better than CPOFDM with OFDM/OQAM for preamble-based channel estimation," in 2008 IEEE International Conference on Communications, pp. 1302-1306, Beijing, China, May 2008.

[15] P. Singh, H. B. Mishra, A. K. Jagannatham, and K. Vasudevan, "Semi-blind, training, and data-aided channel estimation schemes for MIMO-FBMC-OQAM systems," IEEE Transactions on Signal Processing, vol. 67, no. 18, pp. 4668-4682, 2019.

[16] C. Lélé, P. Siohan, R. Legouable, and J.-P. Javaudin, "Preamblebased channel estimation techniques for OFDM/OQAM over the powerline," in 2007 IEEE International Symposium on Power Line Communications and Its Applications, pp. 59-64, Pisa, Italy, March 2007.

[17] D. Kong, X. Zheng, Y. Zhang, and T. Jiang, "Frame repetition: a solution to imaginary interference cancellation in FBMC/OQAM systems," IEEE Transactions on Signal Processing, vol. 68, pp. 1259-1273, 2020.

[18] P. Liu, S. Jin, T. Jiang, Q. Zhang, and M. Matthaiou, "Pilot power allocation through user grouping in multi-cell massive MIMO systems," IEEE Transactions on Communications, vol. 65, no. 4, pp. 1561-1574, 2017.

[19] D. Kong, X.-G. Xia, P. Liu, and Q. Zhu, "MMSE channel estimation for two-port demodulation reference signals in new radio," Science China Information Sciences, vol. 64, no. 6, 2021.

[20] Y. Zhang, D. Wang, J. Wang, and X. You, “Channel estimation for massive MIMO-OFDM systems by tracking the joint angle-delay subspace," IEEE Access, vol. 4, pp. 10166-10179, 2016.

[21] D. Kong, Y. Xu, G. Song, J. Li, and T. Jiang, “A CP reduction scheme based on symbol repetition for narrow-band IoT systems," IEEE Internet of Things Journal, 2021. 